\section{A iodúria de pacientes portadores de tireopatias autoimunes em Santo André, SP, é comparável à dos indivíduos normais e estável nos últimos dez anos}

\author{
Urinary iodine in patients with auto-immune thyroid \\ disorders in Santo André, SP, is comparable to normal \\ controls and has been steady for the last 10 years
}

Maria Angela Zaccarelli Marino', Lourdes Conceição Martins ${ }^{2,3}$, Roberto Z. Esteves ${ }^{4}$, Teresa S. Kasamatsu4, Rui M. B. Maciel ${ }^{4}$

\begin{abstract}
RESUMO
Objetivo: Avaliar se o aumento de iodo na dieta seria o fator desencadeante de tireopatias autoimunes na cidade de Santo André, SP. Métodos: Determinou-se a iodúria em amostra isolada de 58 pacientes, divididos em quatro grupos e de 13 indivíduos normais (controles). Resultados: lodúria: grupo 1 - hipertireoidismo = 203,5 $\pm 152,71 \mu \mathrm{g} / \mathrm{L}$ (média $\pm \mathrm{DP}$ ); grupo 2 hipotireoidismo $=258,31 \pm 148,2 \mu \mathrm{g} / \mathrm{L} ;$ grupo 3 - tireodite crônica autoimune $=244,29 \pm 191,6$ $\mu \mathrm{g} / \mathrm{L} ;$ grupo 4 - amiodarona = 1.157,5 $\pm 261,8 \mu \mathrm{g} / \mathrm{L} ;$ grupo 5 - controles $=262,31 \pm 146,2 \mu \mathrm{g} / \mathrm{L}$. Comparadas as médias da iodúria entre os cinco grupos, dos grupos 1, 2, 3 e 5 não apresentaram diferenças significantes $(p>0,05)$ e todos diferiram do grupo $4(p<0,05)$. A iodúria dos grupos 1, 2, 3 e 5, obtida em 2002 e 2003, não diferiram dos valores determinados em 1994 em escolares em Santo André. Conclusão: Este estudo evidencia que o iodo não deve ser considerado o agente responsável pelas tireopatias autoimunes em Santo André, e outros fatores ambientais devem ser investigados. Arq Bras Endocrinol Metab. 2009;53(1):55-63.
\end{abstract}

Descritores

Iodúria; tireoidite crônica autoimune; amiodarona; hipotireoidismo; hipertireoidismo

\begin{abstract}
Objective: Evaluate whether the increase of iodine in the diet would be the triggering factor for auto-immune thyropathies in the city of Santo André, SP. Methods: Urinary iodine was determined in samples isolated from 58 patients, divided in 4 Groups, and in 13 normal individuals (controls). Results: Urinary lodine: Group 1 - hyperthyroidism $=203.5 \pm 152.71 \mu \mathrm{g} /$ $\mathrm{L}($ mean $\pm \mathrm{sd})$; Group 2 - hypothyroidism = 258.31 $\pm 148,2 \mu \mathrm{g} / \mathrm{L}$; Group 3 - chronic auto-immune thyroiditis $=244.29 \pm 191.6 \mu \mathrm{g} / \mathrm{L} ;$ group 4 (Amiodarone) $=1157.5 \pm 261.8 \mu \mathrm{g} / \mathrm{L}$; Group 5 - Controls $=262.31 \pm 146.2 \mu \mathrm{g} / \mathrm{L}$. On comparing the means of urinary iodine among the groups, the means for groups $1,2,3$, and 5 did not present significant differences ( $p>0.05$ ), and all differed from group $4(p<0.05)$. Urinary iodine obtained in groups 1, 2, 3 and 5, obtained in 2002-03, is not different from the values determined in students in 1994 in Santo André. Conclusion: This study shows evidence that iodine should not be considered as the responsible agent for auto-immune thyropathies in Santo André, and other environmental factors should be investigated. Arq Bras Endocrinol Metab. 2009;53(1):55-63.
\end{abstract}

Keywords

Urinary iodine; chronic auto-immune thyroiditis; amiodarone; hypothyroidism; hyperthyroidism
Disciplina de Endocrinologia do Departamento de Clínica Médica da Faculdade de Medicina da Fundação ABC 2 Departamento de Saúde da Coletividade da Faculdade de Medicina da Fundação $A B C$ ${ }^{3}$ Laboratório de Poluição Atmosférica Ambiental da Faculdade de Medicina da Universidade de São Paulo (USP) ${ }^{4}$ Disciplina de Endocrinologia do Departamento de Medicina da Escola Paulista de Medicina da Universidade Federal de São Paulo (EPM-Unifesp); São Paulo, SP, Brazil

Correspondência para: Maria Angela Zaccarelli Marino Rua Dr. Gabriel dos Santos, 405 $20^{\circ}$ andar

01231-011São Paulo SP mangelazaccarelli@yahoo.com.br

Recebido em 25/Jun/2008 Aceito em 31/Out/2008 


\section{INTRODUÇÃO}

A s doenças autoimunes da tireóide têm sido diagnosticadas com mais frequência, particularmente a tireoidite crônica autoimune $(1,2)$. Existem dúvidas se este aumento de incidência seria em virtude da melhora dos métodos diagnósticos ou de fatores ambientais relacionados à elevação da ocorrência da moléstia. As tireopatias são encontradas predominantemente no sexo feminino, na faixa etária entre 40 e 60 anos $(2,3)$. A prevalência dos anticorpos antitireoidianos gerados pelo sistema imunológico dos pacientes afetados é muito variada e dependente da idade, do sexo e, possivelmente, da ingestão de iodo $(2,3)$.

A glândula tireóide utiliza o iodo para realizar a síntese hormonal da triiodotironina (T3) e da tiroxina (T4) e a necessidade deste nutriente na dieta é de 150 a 200 $\mu \mathrm{g} /$ dia, visto que quantidades menores que $50 \mu \mathrm{g} / \mathrm{dia}$ levam à carência deste elemento, com consequente hipofunção tireoidiana; por outro lado, o excesso de iodo, isto é, quantidades maiores que $1.000 \mu \mathrm{g} /$ dia podem levar à maior prevalência de doenças autoimunes tireoidianas (4).

As doenças tireoidianas autoimunes são determinadas pela perda da autotolerância imunológica e causadas, possivelmente, pela combinação de múltiplos fatores, genéticos e ambientais, mas a identificação e o papel de cada um deles não estão bem esclarecidos (5-7). Elas pertencem ao grupo de doenças, das quais as mais prevalentes são a tireoidite de Hashimoto e a doença de Basedow-Graves, caracterizadas por infiltrado linfocítico de intensidade variável na tireóide e produção de autoanticorpos tireoidianos dirigidos a antígenos específicos, determinantes da expressão clínica da enfermidade que varia do hipertireoidismo ao hipotireoidismo (8). Outras formas de tireopatias autoimunes incluem a tireoidite pós-parto, a tireoidite silenciosa, a tireoidite induzida por $\alpha$-interferon e a tireoidite que acompanha as síndromes autoimunes poliglandulares (8). A tireoglobulina ( $\mathrm{Tg}$ ), a tireoperoxidase tireoidiana (TPO) e o receptor do TSH (TSHR) são considerados os principais autoantígenos tireoidianos específicos na resposta autoimune tireoidiana e os respectivos anticorpos - anticorpo anti-Tg, anticorpo anti-TPO e anticorpo anti-TSHR - são as ferramentas diagnósticas para o diagnóstico da tireopatias autoimunes, o último patognomônico da doença de Basedow-Graves (8). Nas associações de doenças autoimunes da tireóide, como a tireoidite de Hashimoto e a doença de Basedow-Graves, os anticorpos coexistem, e a disfunção resultante será consequente ao predomínio de um deles, A-Tg e A-TPO ou TSHR.
A concentração de iodo tem sido considerada fator modulador da autoimunidade tireoidiana $(9,10)$. Em geral, sua deficiência atenua, enquanto seu excesso acelera a tireoidite autoimune em indivíduos geneticamente suscetíveis. Estudos epidemiológicos sugerem que as tireopatias autoimunes são mais comuns em áreas com excesso de iodo. Assim, em regiões onde a ingestão de iodo é elevada, como no Japão, a incidência de tireoidite de Hashimoto é maior quando comparada às regiões onde a ingestão de iodo é normal ou relativamente baixa. Do mesmo modo, a doença de Basedow-Graves é mais frequente em áreas repletas de iodo, enquanto causas não-autoimunes de hipertireoidismo são mais prevalentes em regiões com baixo conteúdo de iodo na dieta (8).

O objetivo deste trabalho é demonstrar se o aumento da oferta de iodo na dieta poderia ser o fator desencadeante de tireopatias autoimunes nos últimos anos em regiões de divisa dos municípios de Santo André e São Paulo, estado de São Paulo.

\section{CASUÍSTICA E MÉTODOS}

Foram selecionados 71 pacientes, no período compreendido entre os anos de 2002 e 2003 , que procuraram a Clínica de Endocrinologia de um dos autores (MAZM), situado na cidade de Santo André, estado de São Paulo, porquanto 58 eram portadores de tireopatias autoimunes e $13 \mathrm{sem}$ tireopatias, todos procedentes de regiões de divisa dos municípios de Santo André e São Paulo, SP.

Dos 71 pacientes avaliados, 57 eram do sexo feminino com idades entre 5 e 74 anos e 14 do sexo masculino com idades entre 7 e 63 anos.

Todos os pacientes foram submetidos aos exames hormonais basais séricos de triiodotironina total (T3), tiroxina total (T4), tiroxina livre (T4L), hormônio tireoestimulante (TSH), anticorpo antitireoglobulina (A-Tg), anticorpo antitireoperoxidase (A-TPO), anticorpo antirreceptor do TSH (TSHR) e ultrassonografia da tireóide e, de acordo com estes e com o quadro clínico, classificados em cinco grupos, assim discriminados.

Grupo 1: oito pacientes portadores de hipertireoidismo primário de etiologia autoimune (doença de Basedow-Graves), quatro do sexo masculino e quatro do sexo feminino com idades que variavam entre 22 e 43 anos. Os critérios diagnósticos foram os sinais e os sintomas clínicos, os valores de T3, T4 e T4L elevados, TSH suprimido, TSHR positivo e presença de bócio confirmada pela ultrassonografia da tireóide.

Grupo 2: trinta e nove pacientes portadores de hipotireoidismo primário de etiologia autoimune, 32 do sexo 
feminino e sete do sexo masculino, com idades que variavam entre 5 e 74 anos. Os critérios diagnósticos foram os sinais e sintomas clínicos, valores do T4L diminuídos e TSH igual ou maior que $10 \mathrm{mU} / \mathrm{L}$ e a presença do A-Tg e do A-TPO, que determinou a origem autoimune.

Grupo 3: sete pacientes portadores de tireoidite crônica autoimune, todos eram do sexo feminino com idades que variavam entre 27 e 65 anos. Os critérios diagnósticos foram T3, T4, T4L e TSH normais e A-Tg e de A-TPO positivos e a presença de bócio determinou a tireoidite de Hashimoto.

Grupo 4: grupo amiodarona, constituído por quatro pacientes portadores de hipotireoidismo primário de etiologia autoimune que faziam uso de amiodarona, todos eram do sexo feminino, com idades que variavam entre 26 e 63 anos.

Grupo 5: grupo-controle, constituído por 13 indivíduos sem tireopatias, dez eram do sexo feminino e três do sexo masculino, com idades que variavam entre 22 e 63 anos. Os critérios de avaliação para a ausência de tireopatias foram T3, T4, T4L e TSH normais, A-Tg, A-TPO e TSHR negativos e ultrassonografia da tireóide normal. Estes indivíduos procuraram o consultório médico relatando sintomas de doenças tireoidianas, que foram afastadas após os exames complementares.

Todos os pacientes dos grupos 1, 2, 3 e 5 não faziam uso de quaisquer medicamentos que pudessem interferir neste estudo, e o uso de substâncias e exames radiológicos com a presença de iodo foram diretamente investigados; somente os pacientes do grupo 4 faziam uso da amiodarona.

Após a confirmação diagnóstica das tireopatias autoimunes e também dos indivíduos sem tireopatias, os 71 pacientes foram submetidos às coletas de urina. As amostras de urina permaneceram congeladas em tubos de ensaio até a realização das dosagens do iodo urinário (iodúria).

As dosagens do T3 total, T4 total, T4 livre, TSH, A-Tg e A-TPO foram realizadas no soro pela técnica de quimiluminescência (11). Foram considerados normais os valores de T3 total de 60 a $210 \mathrm{ng} / \mathrm{dL}$ para adultos e de 94 a $241 \mathrm{ng} / \mathrm{dL}$ para aqueles com 5 a 12 anos de idade; de T4 total de 4,5 a $13,5 \mu \mathrm{g} / \mathrm{dL}$ para adultos e de 6,0 a $14,0 \mu \mathrm{g} / \mathrm{dL}$ para aqueles com 5 a 10 anos de idade; T4 livre de 0,8 a $1,9 \mathrm{ng} / \mathrm{mL}$ e TSH de 0,4 a 4,0 $\mathrm{mU} / \mathrm{L}$. Foram considerados negativos os valores de A-Tg e A-TPO inferiores a $40 \mathrm{UI} / \mathrm{mL}$ e $35 \mathrm{UI} / \mathrm{mL}$, respectivamente. As dosagens do anticorpo anti-receptor do TSH (TSHR) foram realizadas pelo método de radioimunoensaio (radiorreceptor), sendo considerados positivos inibição maior que $10 \%$.
As dosagens da iodúria foram realizadas por método semiautomatizado precedido por digestão leve com persulfato de amônio que detecta o iodo pela monitoração da redução do sulfato cérico amoniacal (12-14). Os valores são expressos em microgramas por litro $(\mu \mathrm{g} / \mathrm{L})$ de urina e classificados, de acordo com o International Council for Control of Iodine Deficiency Disorders (ICCIDD), em deficiência grave de iodo quando a mediana de iodúria é menor que $25 \mu \mathrm{g} / \mathrm{L}$; deficiência moderada quando a mediana está entre $25 \mu \mathrm{g} / \mathrm{L}$ e $49 \mu \mathrm{g} / \mathrm{L}$; deficiência leve quando a mediana está entre $50 \mu \mathrm{g} / \mathrm{L}$ e $99 \mu \mathrm{g} / \mathrm{L}$; e normal ou suficiência de iodo quando a mediana se apresenta acima de $100 \mu \mathrm{g} / \mathrm{L}(14,15)$.

A ultrassonografia da tireóide foi realizada com transdutor linear multifrequencial de alta resolução, 7,5 MHz e $10 \mathrm{MHz}$ com volume normal de 8,3 a $17,1 \mathrm{~mL}$.

A análise estatística foi realizada pelo teste de KruskalWallis para comparação das médias de iodo urinário entre os cinco grupos e pelo teste de comparações múltiplas de Turkey. O nível de significância é de $5 \%(\mathrm{p}<0,05)$.

Foi explicado a todos os pacientes os objetivos e os métodos do trabalho. Todos concordaram com ele e assinaram seu consentimento livre e esclarecido.

O protocolo deste trabalho foi aprovado pelo Comitê de Ética em Pesquisa da Faculdade de Medicina do ABC, registrado sob o $\mathrm{n}^{\mathrm{O}} 284 / 2007$.

Foram incluídos neste trabalho, para comparação com a iodúria dos pacientes estudados, os valores de iodúria obtidos há cerca de dez anos (1994) em 40 escolares, de 8 a 10 anos, moradores em Santo André, SP. Estes valores foram obtidos durante o $3^{\circ}$ Inquérito Nacional de Recenseamento da Endemia de Carência de Iodo no Brasil, patrocinado pelo Ministério da Saúde e pelo Fundo das Nações Unidas para a Infância (Unicef), e dosados no Laboratório de Poluição Atmosférica Ambiental da Faculdade de Medicina da Universidade de São Paulo (FM-USP), de acordo com a mesma metodologia empregada no estudo atual $(12,14)$. Para o inquérito de 1994 , adotou-se o sistema de amostragem híbrido que abrangia 13 municípios sentinelas de deficiência de iodo, nos quais havia sido evidenciada maior prevalência de bócio em 1990, os municípios limítrofes a estes primeiros e municípios escolhidos aleatoriamente, a fim de obter amostra representativa de cada unidade da Federação. Santo André foi um dos 15 municípios escolhidos no estado de São Paulo, em conjunto com Assis, Bebedouro, Francisco Morato, Itanhaém, Jacareí, Osasco, Piracicaba, Santa Fé do Sul, Santos, São Paulo, Sarapuí, Serra Negra, Sumaré e Taboão da Serra $(12,16)$. 


\section{RESULTADOS}

Dos 71 indivíduos avaliados, $58(81,70 \%)$ apresentavam tireopatias autoimunes e $13(18,30 \%)$ foram considerados sem tireopatias e pertencentes ao grupo-controle (grupo 5).

Dos 58 pacientes com tireopatias autoimunes, 47 $(81,03 \%)$ eram do sexo feminino, com idades entre 5 e 74 anos e 11 (18,97\%) do sexo masculino, com idades entre 7 e 53 anos. Dos 13 indivíduos do grupo-controle, dez $(76,93 \%)$ eram do sexo feminino, com idades entre 22 e 56 anos e três $(23,07 \%)$ do sexo masculino, com idades entre 37 e 63 anos.

Dos 58 pacientes portadores de tireopatias autoimunes, oito (13,80\%) do grupo 1 apresentavam hipertireoidismo primário; $39(67,24 \%)$ do grupo 2 , com hipotireoidismo primário, 22 destes $(56,41 \%)$ apresentavam tireoidite crônica autoimune e $17(43,59 \%)$ tireoidite de Hashimoto; sete $(12,06 \%)$ do grupo 3 com tireoidite crônica autoimune $(42,86 \%)$, sendo quatro $(57,14 \%)$ com tireoidite de Hashimoto; quatro $(6,90 \%)$ do grupo 4 com hipotireoidismo primário faziam uso da amiodarona, visto três destes $(75 \%)$ apresentavam tireoidite crônica autoimune e um (25\%) tireoidite de Hashimoto.

Os valores séricos basais e os valores médios séricos basais do T3 total, T4 total, T4L, TSH, A-Tg, A-TPO, TSHR, iodúria, assim como os dados da ultrassonografia da tireóide dos grupos 1, 2, 3 e 4 estão demonstrados nas Tabelas 1, 2, 3 e 4, os do grupo-controle na Tabela 5 .

Os valores médios do volume da tireóide, nos pacientes com hipertireoidismo primário foram de $21,0 \mathrm{~mL}$; nos pacientes com hipotireoidismo primário foram de 19,4 mL; e nos pacientes com tireoidite de Hashimoto foram de 18,1 mL.
A descrição dos valores de tendência central e dispersão por grupo de estudo da iodúria está demonstrada na Tabela 6. Quando foram comparadas as médias da iodúria entre os cinco grupos, pode-se observar que havia diferença significativa entre eles $(\mathrm{p}<0,05)$. Quando foram comparadas as médias da iodúria dos grupos do hipertireoidismo primário, hipotireoidismo primário, tireoidite crônica autoimune e controle, pode-se observar que diferem do grupo amiodarona $(\mathrm{p}<0,05)$. Se o grupo amiodarona for excluído, não serão observadas diferenças significativas nas médias da iodúria entre os demais grupos $(\mathrm{p}>0,05)$.

Em relação à iodúria obtida em 1994, por ocasião do $3^{\circ}$ Inquérito Nacional de Recenseamento da Endemia de Carência de Iodo no Brasil, houve a coleta de urina de 40 escolares de Santo André, SP, que apresentaram a seguinte distribuição: a) deficiência grave $(<25$ $\mu \mathrm{g} / \mathrm{L})$ : zero escolares $(0 \%) ; \mathrm{b})$ deficiência moderada (entre $\leq 25 \mu \mathrm{g} / \mathrm{L} \mathrm{e}<50 \mu \mathrm{g} / \mathrm{L})$ : um escolar $(2,5 \%)$; c) deficiência leve $($ entre $\leq 50 \mu \mathrm{g} / \mathrm{L}$ e $100 \mu \mathrm{g} / \mathrm{L})$ : seis escolares (15\%); d) suficiência (entre $100 \mu \mathrm{g} / \mathrm{L}$ e 200 $\mu \mathrm{g} / \mathrm{L}): 22$ escolares (55\%); e) mais do que suficiência (acima de $200 \mu \mathrm{g} / \mathrm{L}$ ): 11 escolares $(27,5 \%)$. A presença de mais de $80 \%$ dos escolares com valores de iodúria suficientes e mais do que suficientes colocava Santo André, SP, entre as cidades com melhor aporte de iodo no país e comparável à maioria das cidades do estado de São Paulo, que foi considerado, no geral, como região de aporte suficiente de iodo, diferentemente de outros estados da Federação, que apresentou alguns municípios com aporte considerado com deficiência leve ou moderada (12).

Tabela 1. Hipertireoidismo primário - grupo 1 - valores de iodo urinário, valores séricos basais de T3 total, T4 total, T4L, TSH, A-Tg, A-TP0 e TSHR. Dados da ultrassonografia da tireóide (UST).

\begin{tabular}{|c|c|c|c|c|c|c|c|c|c|c|c|c|c|c|}
\hline \multirow[t]{2}{*}{ Pacientes } & \multirow[t]{2}{*}{ Sexo } & \multirow{2}{*}{$\begin{array}{l}\text { Idade } \\
\text { (anos) }\end{array}$} & \multirow{2}{*}{$\begin{array}{l}\text { lodo } \\
(\mu \mathrm{g} / \mathrm{L})\end{array}$} & \multirow{2}{*}{$\begin{array}{c}\text { T3 } \\
(\mathrm{ng} / 100 \mathrm{~mL})\end{array}$} & \multirow{2}{*}{$\begin{array}{c}\mathrm{T4} \\
(\mu \mathrm{g} / / \mathrm{dL})\end{array}$} & \multirow{2}{*}{$\begin{array}{c}\mathrm{T} 4 \mathrm{~L} \\
(\mathrm{ng} / 100 \mathrm{~mL})\end{array}$} & \multirow{2}{*}{$\begin{array}{c}\text { TSH } \\
(\mu \mathrm{Ul} / \mathrm{mL})\end{array}$} & \multirow{2}{*}{$\begin{array}{c}A-\mathrm{Tg} \\
(\mathrm{Ul} / \mathrm{mL})\end{array}$} & \multirow{2}{*}{$\begin{array}{c}\text { A-TPO } \\
\text { (UI/mL) }\end{array}$} & \multirow{2}{*}{$\begin{array}{c}\text { TSHR } \\
\%\end{array}$} & \multicolumn{2}{|c|}{ UST (cm) } & \multicolumn{2}{|c|}{ Nódulo (cm) } \\
\hline & & & & & & & & & & & Direito & Esquerdo & Direito & Esquerdo \\
\hline 1 & M & 43 & 430,00 & 327,00 & 16,3 & 2,6 & 0,05 & - & - & 30 & $6,3 \times 2,9 \times 1,1$ & $5,6 \times 2,8 \times 1,0$ & - & - \\
\hline 3 & M & 25 & 40,00 & 299,00 & 17,1 & 2,1 & 0,01 & 100 & 352 & 24 & $4,8 \times 1,4 \times 1,2$ & $4,3 \times 1,8 \times 1,2$ & - & 1,1 \\
\hline 4 & M & 22 & 109,00 & 443,00 & 25,3 & 4,6 & 0,01 & - & - & 54 & $5,0 \times 1,9 \times 2,1$ & $4,6 \times 1,6 \times 2,0$ & 0,5 & - \\
\hline 6 & $\mathrm{~F}$ & 31 & 365,00 & 371,00 & 17,2 & 3,6 & 0,05 & - & 110 & 22 & $8,2 \times 3,2 \times 2,2$ & $4,9 \times 1,9 \times 1,2$ & - & - \\
\hline 7 & M & 40 & 107,00 & 565,00 & 28,9 & 4,6 & 0,01 & 100 & 854 & 44 & $5,0 \times 2,2 \times 1,1$ & $4,2 \times 2,1 \times 1,2$ & 0,5 & - \\
\hline 8 & $F$ & 39 & 42,00 & 247,00 & 18,1 & 2,6 & 0,05 & - & - & 15 & $6,2 \times 2,2 \times 1,3$ & $4,1 \times 2,1 \times 1,1$ & - & - \\
\hline Média & & 34 & 203,50 & 378,13 & 20,34 & 3,30 & 0,03 & 86,67 & 369,00 & 38,25 & & & & \\
\hline
\end{tabular}

T3: triiodotironina; T4; tiroxina; T4L: tiroxina livre; TSH: hormônio tireoestimulante; A-Tg: anticorpo antitireoglobulina; A-TPO: anticorpo antitireoperoxidase; TSHR: anticorpo anti-receptor do TSH. 
Tabela 2. Hipotireoidismo primário - grupo 2 - valores de iodo urinário, valores séricos basais de T3 total, T4 total, T4L, TSH, A-Tg, A-TP0. Dados da ultrassonografia da tireóide (UST).

\begin{tabular}{|c|c|c|c|c|c|c|c|c|c|c|c|c|c|}
\hline \multirow[t]{2}{*}{ Pacientes } & \multirow[t]{2}{*}{ Sexo } & \multirow{2}{*}{$\begin{array}{l}\text { Idade } \\
\text { (anos) }\end{array}$} & \multirow{2}{*}{$\begin{array}{l}\text { lodo } \\
(\mu \mathrm{g} / \mathrm{L})\end{array}$} & \multirow{2}{*}{$\begin{array}{c}\text { T3 } \\
\text { (ng/100mL) }\end{array}$} & \multirow{2}{*}{$\begin{array}{c}\mathrm{T} 4 \\
(\mu \mathrm{g} / / \mathrm{dL})\end{array}$} & \multirow{2}{*}{$\begin{array}{c}\mathrm{T} 4 \mathrm{~L} \\
(\mathrm{ng} / 100 \mathrm{~mL})\end{array}$} & \multirow{2}{*}{$\begin{array}{c}\text { TSH } \\
(\mu \mathrm{Ul} / \mathrm{mL})\end{array}$} & \multirow{2}{*}{$\begin{array}{c}\mathrm{A}-\mathrm{Tg} \\
(\mathrm{Ul} / \mathrm{mL})\end{array}$} & \multirow{2}{*}{$\begin{array}{l}\text { A-TPO } \\
\text { (UI/mL) }\end{array}$} & \multicolumn{2}{|c|}{ UST(cm) } & \multicolumn{2}{|c|}{ Nódulo(cm) } \\
\hline & & & & & & & & & & Direito & Esquerdo & Direito & Esquerdo \\
\hline 1 & $\mathrm{~F}$ & 42 & 450,0 & 85,0 & 2,1 & 0,1 & 129,0 & 1600 & 600 & $3,0 \times 2,1 \times 1,4$ & $4,2 \times 1,1 \times 1,3$ & - & - \\
\hline 2 & $\mathrm{~F}$ & 12 & 148,0 & 90,0 & 6,4 & 0,6 & 23,5 & 600 & 1600 & $3,0 \times 2,2 \times 1,1$ & $3,2 \times 2,1 \times 1,2$ & 0,5 & - \\
\hline 3 & M & 7 & 270,0 & 80,0 & 6,1 & 0,7 & 25,0 & 292 & 548 & $2,2 \times 1,2 \times 1,3$ & $2,1 \times 1,1 \times 1,1$ & - & - \\
\hline 4 & $\mathrm{~F}$ & 51 & 204,0 & 84,0 & 4,5 & 0,6 & 22,0 & 3000 & 500 & $1,7 \times 0,6 \times 0,8$ & $1,2 \times 0,6 \times 0,0$ & 0,8 & - \\
\hline 5 & $\mathrm{~F}$ & 50 & 575,0 & 85,0 & 5,5 & 0,5 & 22,4 & 250 & 100 & $5,4 \times 1,8 \times 1,6$ & $4,5 \times 2,0 \times 1,4$ & - & - \\
\hline 6 & M & 49 & 129,0 & 63,0 & 5,8 & 0,5 & 20,0 & 3000 & 400 & $4,4 \times 1,5 \times 1,3$ & $4,1 \times 1,0 \times 1,3$ & - & - \\
\hline 7 & $\mathrm{~F}$ & 21 & 136,0 & 62,0 & 2,5 & 0,2 & 27,6 & 100 & 400 & $4,0 \times 1,3 \times 1,3$ & $4,0 \times 1,2 \times 1,2$ & - & - \\
\hline 8 & M & 46 & 93,0 & 65,0 & 4,5 & 0,5 & 20,4 & 6400 & 25600 & $4,0 \times 2,2 \times 2,2$ & $2,2 \times 1,2 \times 1,1$ & 0,5 & - \\
\hline 9 & $\mathrm{~F}$ & 24 & 530,0 & 32,0 & 0,7 & 0,1 & 298,0 & 800 & 500 & $5,9 \times 1,3 \times 1,8$ & $5,6 \times 1,6 \times 1,7$ & - & - \\
\hline 10 & $\mathrm{~F}$ & 38 & 92,0 & 69,0 & 4,8 & 0,4 & 31,3 & 2000 & 5000 & $5,4 \times 2,5 \times 2,1$ & $4,4 \times 2,0 \times 1,1$ & 0,5 & - \\
\hline 11 & $\mathrm{~F}$ & 15 & 590,0 & 63,0 & 4,4 & 0,5 & 17,0 & 6400 & 600 & $4,4 \times 1,4 \times 4,3$ & $4,3 \times 1,4 \times 1,5$ & 1,9 & - \\
\hline 12 & $\mathrm{~F}$ & 34 & 230,0 & 84,0 & 5,9 & 0,4 & 20,0 & 3000 & 3000 & $4,9 \times 1,7 \times 1,4$ & $4,5 \times 1,3 \times 1,4$ & - & - \\
\hline 13 & $\mathrm{~F}$ & 27 & 110,0 & 63,0 & 3,7 & 0,1 & 109,0 & 163 & 240 & $4,7 \times 1,6 \times 1,3$ & $4,5 \times 1,2 \times 1,2$ & - & - \\
\hline 14 & $\mathrm{~F}$ & 13 & 260,0 & 94,0 & 5,6 & 0,6 & 28,2 & 822 & 2692 & $4,8 \times 1,6 \times 1,6$ & $4,4 \times 1,6 \times 1,7$ & - & - \\
\hline 15 & $\mathrm{~F}$ & 9 & 375,0 & 48,0 & 3,9 & 0,5 & 40,1 & 694 & 779 & $4,1 \times 1,5 \times 1,5$ & $4,1 \times 1,7 X, 1,7$ & - & - \\
\hline 16 & $\mathrm{~F}$ & 21 & 240,0 & 68,0 & 5,4 & 0,6 & 17,9 & 580 & 580 & $4,4 \times 1,1 \times 1,1$ & $3,9 \times 1,0 \times 0,9$ & - & - \\
\hline 17 & $\mathrm{~F}$ & 42 & 430,0 & 55,0 & 0,5 & 0,4 & 135,0 & 320 & 405 & $2,8 \times 1,0 \times 0,9$ & $2,4 \times 1,2 \times 0,9$ & 0,7 & - \\
\hline 18 & $\mathrm{~F}$ & 49 & 187,0 & 98,0 & 5,3 & 0,5 & 19,7 & 434 & 152 & $4,0 \times 1,0 \times 1,3$ & $4,0 \times 1,0 \times 1,3$ & 0,9 & 0,8 \\
\hline 19 & $\mathrm{~F}$ & 36 & 253,0 & 43,0 & 2,2 & 0,4 & 68,0 & 1600 & 6400 & $3,6 \times 1,4 \times 1,4$ & $3,7 \times 1,2 \times 1,1$ & 0,7 & - \\
\hline 20 & $\mathrm{~F}$ & 5 & 275,0 & 69,0 & 4,1 & 0,6 & 16,1 & 400 & 400 & $3,3 \times 1,0 \times 1,0$ & $3,6 \times 1,1 \times 1,2$ & - & - \\
\hline 21 & $\mathrm{~F}$ & 44 & 193,0 & 90,0 & 8,2 & 0,2 & 84,2 & 600 & 200 & $4,8 \times 2,1 \times 1,7$ & $4,4 \times 1,8 \times 1,2$ & - & - \\
\hline 22 & $\mathrm{~F}$ & 45 & 88,0 & 89,0 & 3,8 & 0,4 & 90,0 & 253 & 489 & $3,2 \times 1,9 \times 2,2$ & $3,6 \times 1,9 \times 1,7$ & - & - \\
\hline 23 & $\mathrm{~F}$ & 31 & 582,0 & 76,0 & 4,8 & 0,6 & 17,7 & 400 & 1600 & $4,7 \times 1,7 \times 1,9$ & $4,3 \times 1,5 \times 1,3$ & - & 0,8 \\
\hline 24 & $F$ & 39 & 286,0 & 75,0 & 6,3 & 0,5 & 15,6 & 400 & 400 & $3,6 \times 1,4 \times 1,1$ & $3,6 \times 1,1 \times 1,8$ & - & - \\
\hline 25 & $\mathrm{~F}$ & 74 & 475,0 & 98,0 & 4,9 & 0,5 & 17,1 & 518 & 840 & $5,3 \times 2,0 \times 1,8$ & $4,2 \times 1,5 \times 1,3$ & 1,7 & - \\
\hline 26 & $\mathrm{~F}$ & 37 & 113,0 & 89,0 & 1,2 & 0,1 & 16,1 & 1000 & 569 & $4,4 \times 1,4 \times 1,9$ & $4,1 \times 1,2 \times 1,8$ & - & - \\
\hline 27 & $\mathrm{~F}$ & 51 & 280,0 & 93,0 & 5,3 & 0,2 & 27,3 & 71 & 701 & $4,5 \times 2,1 \times 2,4$ & $3,8 \times 1,9 \times 1,1$ & 0,6 & 5,0 \\
\hline 28 & $\mathrm{~F}$ & 34 & 375,0 & 50,0 & 2,9 & 0,1 & 164,0 & 714 & 840 & $2,6 \times 0,9 \times 0,9$ & $3,1 \times 1,3 \times 1,2$ & - & - \\
\hline 29 & M & 51 & 260,0 & 85,0 & 4,8 & 0,4 & 32,0 & 2796 & 169 & $5,8 \times 3,4 \times 1,8$ & $4,5 \times 2,6 \times 1,7$ & 0,8 & 5,5 \\
\hline 30 & $\mathrm{~F}$ & 56 & 113,0 & 72,0 & 4,6 & 0,4 & 19,7 & 1178 & 434 & $3,2 \times 1,3 \times 1,0$ & $3,2 \times 1,0 \times 0,9$ & - & - \\
\hline 31 & M & 53 & 264,0 & 83,0 & 3,0 & 0,4 & 66,0 & 2000 & 1934 & $5,7 \times 1,9 \times 2,1$ & $4,1 \times 1,8 \times 18$ & 1,8 & - \\
\hline 32 & M & 43 & 152,0 & 88,0 & 3,5 & 0,4 & 59,0 & 503 & 424 & $4,2 \times 2,9 \times 1,5$ & $4,7 \times 1,6 \times 1,2$ & 1,5 & - \\
\hline 33 & $\mathrm{~F}$ & 43 & 51,0 & 78,0 & 5,8 & 0,4 & 17,6 & 624 & 248 & $3,3 \times 1,4 \times 1,4$ & $3,7 \times 1,5 \times 1,4$ & 0,5 & - \\
\hline 34 & $\mathrm{~F}$ & 50 & 151,0 & 98,0 & 6,9 & 0,5 & 17,8 & 448 & 840 & $3,2 \times 1,4 \times 1,2$ & $3,4 \times 1,3 \times 1,1$ & - & - \\
\hline 35 & $\mathrm{~F}$ & 29 & 240,0 & 68,0 & 4,2 & 0,5 & 17,2 & 400 & 400 & $2,2 \times 2,8 \times 4,7$ & $1,2 \times 1,6 \times 4,7$ & 0,6 & - \\
\hline 36 & $\mathrm{~F}$ & 33 & 330,0 & 98,0 & 5,4 & 0,2 & 24,0 & 1788 & 3000 & $3,6 \times 1,1 \times 1,2$ & $3,7 \times 1,4 \times 1,2$ & - & - \\
\hline 37 & $\mathrm{~F}$ & 28 & 106,0 & 84,0 & 4,8 & 0,5 & 86,0 & 1000 & 3000 & $4,8 \times 2,0 \times 1,4$ & $4,7 \times 2,0 \times 1,4$ & - & - \\
\hline 38 & $\mathrm{~F}$ & 58 & 220,0 & 89,0 & 5,9 & 0,4 & 19,0 & 600 & 600 & $2,9 \times 1,1 \times 1,1$ & $1,9 \times 1,1 \times 0,7$ & - & - \\
\hline 39 & M & 44 & 218,0 & 62,0 & 6,1 & 0,4 & 50,0 & 400 & 350 & $6,0 \times 4,0 \times 3,0$ & $5,5 \times 4,5 \times 2,0$ & 0,6 & 0,1 \\
\hline Média & & 37 & 258,31 & 76,03 & 4,52 & 0,41 & 49,5 & 1234,56 & 1731,64 & & & & \\
\hline
\end{tabular}

T3: triiodotironina; T4;tiroxina; T4L: tiroxinalivre; TSH: hormônio tireoestimulante; A-Tg: anticorpo antitireoglobulina; A-TPO: anticorpo antitireoperoxidase. 
Tabela 3. Tireoidite crônica autoimune - grupo 3 - valores de iodo urinário, valores sérico basais de T3 total, T4 total, T4L, TSH, A-Tg, A-TPO. Dados da ultrassonografia da tireóide (UST).

\begin{tabular}{|c|c|c|c|c|c|c|c|c|c|c|c|c|c|}
\hline \multirow[t]{2}{*}{ Pacientes } & \multirow[t]{2}{*}{ Sexo } & \multirow{2}{*}{$\begin{array}{l}\text { Idade } \\
\text { (anos) }\end{array}$} & \multirow{2}{*}{$\begin{array}{l}\text { lodo } \\
(\mu \mathrm{g} / \mathrm{L})\end{array}$} & \multirow{2}{*}{$\begin{array}{c}\text { T3 } \\
\text { (ng/100mL) }\end{array}$} & \multirow{2}{*}{$\begin{array}{c}\mathrm{T4} \\
(\mu \mathrm{g} / / \mathrm{dL})\end{array}$} & \multirow{2}{*}{$\begin{array}{c}\text { T4L } \\
(\mathrm{ng} / 100 \mathrm{~mL})\end{array}$} & \multirow{2}{*}{$\begin{array}{c}\text { TSH } \\
(\mu \mathrm{Ul} / \mathrm{mL})\end{array}$} & \multirow{2}{*}{$\begin{array}{c}\text { A-Tg } \\
(\mathrm{Ul} / \mathrm{mL})\end{array}$} & \multirow{2}{*}{$\begin{array}{c}\text { A-TPO } \\
\text { (Ul/mL) }\end{array}$} & \multicolumn{2}{|c|}{ UST (cm) } & \multicolumn{2}{|c|}{ Nódulo (cm) } \\
\hline & & & & & & & & & & Direito & Esquerdo & Direito & Esquerdo \\
\hline 1 & $\mathrm{~F}$ & 51 & 109,0 & 133,0 & 8,9 & 1,4 & 1,2 & 454 & 401 & $4,3 \times 1,9 \times 1,1$ & $3,6 \times 1,8 \times 1,0$ & - & - \\
\hline 2 & $\mathrm{~F}$ & 44 & 300,0 & 122,0 & 9,6 & 0,9 & 2,2 & 282 & 157 & $4,0 \times 1,6 \times 2,0$ & $3,8 \times 1,4 \times 1,7$ & 0,8 & 1,0 \\
\hline 3 & $\mathrm{~F}$ & 32 & 570,0 & 128,0 & 8,2 & 0,9 & 2,9 & 3000 & 1000 & $3,8 \times 1,4 \times 1,2$ & $4,3 \times 1,8 \times 1,2$ & - & 1,4 \\
\hline 4 & $\mathrm{~F}$ & 32 & 63,0 & 133,0 & 8,4 & 1,6 & 3,0 & 160 & 400 & $5,0 \times 1,9 \times 2,1$ & $4,6 \times 1,6 \times 2,0$ & 0,5 & - \\
\hline 5 & $\mathrm{~F}$ & 65 & 420,0 & 120,0 & 9,4 & 1,4 & 2,9 & 250 & 1600 & $4,0 \times 1,1 \times 1,3$ & $4,2 \times 1,1 \times 1,5$ & - & - \\
\hline 6 & $\mathrm{~F}$ & 55 & 123,0 & 105,0 & 8,6 & 1,6 & 0,4 & 240 & 500 & $3,2 \times 2,2 \times 1,2$ & $2,9 \times 1,9 \times 1,2$ & - & - \\
\hline 7 & $\mathrm{~F}$ & 27 & 125,0 & 113,0 & 8,7 & 1,2 & 1,4 & 163 & 240 & $6,0 \times 2,2 \times 1,1$ & $5,2 \times 2,1 \times 1,2$ & 0,5 & - \\
\hline Média & & 44 & 244,29 & 122,00 & 8,83 & 1,29 & 2,00 & 649,86 & 614,00 & & & & \\
\hline
\end{tabular}

T3: triiodotironina; T4: tiroxina; T4L: tiroxina livre; TSH: hormônio tireoestimulante; A-Tg: anticorpo antitireoglobulina; A-TPO: anticorpo antitireoperoxidase.

Tabela 4. Hipotireoidismo primário - grupo 4 - grupo amiodarona - valores de iodo urinário, valores sérico basais de T3 total, T4 total, T4L, TSH, A-Tg, A-TPO. Dados da ultrassonografia da tireóide (UST).

\begin{tabular}{|c|c|c|c|c|c|c|c|c|c|c|c|c|c|}
\hline \multirow[t]{2}{*}{ Pacientes } & \multirow[t]{2}{*}{ Sexo } & \multirow{2}{*}{$\begin{array}{l}\text { Idade } \\
\text { (anos) }\end{array}$} & \multirow{2}{*}{$\begin{array}{l}\text { lodo } \\
(\mu \mathrm{g} / \mathrm{L})\end{array}$} & \multirow{2}{*}{$\begin{array}{c}\mathrm{T} 3 \\
\text { (ng/100mL) }\end{array}$} & \multirow{2}{*}{$\begin{array}{c}\mathrm{T} 4 \\
(\mu \mathrm{g} / / \mathrm{dL})\end{array}$} & \multirow{2}{*}{$\begin{array}{c}\mathrm{T} 4 \mathrm{~L} \\
(\mathrm{ng} / 100 \mathrm{~mL})\end{array}$} & \multirow{2}{*}{$\begin{array}{l}\text { TSH } \\
(\mu \mathrm{Ul} / \mathrm{mL})\end{array}$} & \multirow{2}{*}{$\begin{array}{l}A-\mathrm{Tg} \\
(\mathrm{UI} / \mathrm{mL})\end{array}$} & \multirow{2}{*}{$\begin{array}{l}\text { A-TPO } \\
\text { (Ul/mL) }\end{array}$} & \multicolumn{2}{|c|}{ UST (cm) } & \multicolumn{2}{|c|}{ Nódulo (cm) } \\
\hline & & & & & & & & & & Direito & Esquerdo & Direito & Esquerdo \\
\hline 1 & $\mathrm{~F}$ & 63 & 1500,0 & 72,0 & 4,4 & 0,5 & 28,7 & 3000 & 500 & $3,9 \times 1,2 \times 1,0$ & $2,8 \times 1,0 \times 9,0$ & - & - \\
\hline 2 & $\mathrm{~F}$ & 52 & 1220,0 & 79,0 & 6,9 & 0,4 & 22,4 & 127 & 254 & $4,1 \times 1,3 \times 1,3$ & $3,2 \times 1,0 \times 1,0$ & 1,6 & - \\
\hline 3 & $\mathrm{~F}$ & 31 & 990,0 & 77,0 & 6,3 & 0,4 & 18,8 & 600 & 400 & $4,2 \times 2,3 \times 2,2$ & $3,4 \times 1,8 \times 1,5$ & - & - \\
\hline 4 & $\mathrm{~F}$ & 26 & 920,0 & 82,0 & 4,8 & 0,5 & 20,4 & 250 & 1600 & $4,4 \times 1,3 \times 1,2$ & $4,0 \times 1,5 \times 1,1$ & - & - \\
\hline Média & & 43 & 1157,50 & 77,50 & 5,60 & 0,45 & 22,58 & 994,25 & 688,50 & & & & \\
\hline
\end{tabular}

T3: triiodotironina; T4; tiroxina; T4L: tiroxina livre; TSH: hormônio tireoestimulante; A-Tg: anticorpo antitireoglobulina; A-TPO: anticorpo antitireoperoxidase.

Tabela 5. Grupo-controle - grupo 5 - valores de iodo urinário, valores sérico basais de T3 total, T4 total, T4L, TSH, A-Tg, A-TP0 e TSHR. Dados da ultrassonografia da tireóide (UST).

\begin{tabular}{|c|c|c|c|c|c|c|c|c|c|c|c|c|c|c|}
\hline \multirow[t]{2}{*}{ Pacientes } & \multirow[t]{2}{*}{ Sexo } & \multirow{2}{*}{$\begin{array}{l}\text { Idade } \\
\text { (anos) }\end{array}$} & \multirow{2}{*}{$\begin{array}{l}\text { lodo } \\
(\mu g / L)\end{array}$} & \multirow{2}{*}{$\begin{array}{c}\mathrm{T} 3 \\
(\mathrm{ng} / 100 \mathrm{~mL})\end{array}$} & \multirow{2}{*}{$\begin{array}{c}\mathrm{T4} \\
(\mu \mathrm{g} / / \mathrm{dL})\end{array}$} & \multirow{2}{*}{$\begin{array}{c}\mathrm{T} 4 \mathrm{~L} \\
\text { (ng/100mL) }\end{array}$} & \multirow{2}{*}{$\begin{array}{c}\text { TSH } \\
(\mu \mathrm{Ul} / \mathrm{mL})\end{array}$} & \multirow{2}{*}{$\begin{array}{c}\mathrm{A}-\mathrm{Tg} \\
(\mathrm{UI} / \mathrm{mL})\end{array}$} & \multirow{2}{*}{$\begin{array}{c}\text { A-TPO } \\
\text { (UI/mL) }\end{array}$} & \multirow{2}{*}{$\begin{array}{c}\text { TSHR } \\
\%\end{array}$} & \multicolumn{2}{|c|}{ UST (cm) } & \multicolumn{2}{|c|}{ Nódulo (cm) } \\
\hline & & & & & & & & & & & Direito & Esquerdo & Direito & Esquerdo \\
\hline 1 & $\mathrm{~F}$ & 29 & 295,0 & 98,0 & 8,4 & 1,3 & 2,7 & Negativo & Negativo & Negativo & $4,3 \times 1,9 \times 1,1$ & $3,6 \times, 8 \times 1,0$ & - & - \\
\hline 2 & M & 40 & 450,0 & 98,0 & 8,4 & 1,6 & 2,2 & Negativo & Negativo & Negativo & $4,0 \times 1,6 \times 2,0$ & $3,8 \times 1,4 \times 1,7$ & - & - \\
\hline 3 & $F$ & 42 & 360,0 & 136,0 & 8,7 & 1,0 & 1,2 & Negativo & Negativo & Negativo & $3,8 \times 1,4 \times 1,2$ & $4,3 \times 1,8 \times 1,2$ & - & - \\
\hline 4 & $F$ & 38 & 150,0 & 95,0 & 7,7 & 0,9 & 1,1 & Negativo & Negativo & Negativo & $3,0 \times 1,9 \times 2,1$ & $3,6 \times 1,6 \times 2,0$ & - & - \\
\hline 5 & M & 63 & 415,0 & 96,0 & 8,5 & 1,4 & 1,7 & Negativo & Negativo & Negativo & $4,0 \times 1,2 \times 1,4$ & $3,6 \times 1,0 \times 1,1$ & - & - \\
\hline 6 & $\mathrm{~F}$ & 35 & 195,0 & 127,0 & 9,9 & 0,9 & 1,2 & Negativo & Negativo & Negativo & $3,2 \times 2,2 \times 1,2$ & $2,9 \times 1,9 \times 1,2$ & - & - \\
\hline 7 & $F$ & 22 & 60,0 & 125,0 & 8,0 & 1,2 & 1,2 & Negativo & Negativo & Negativo & $3,0 \times 2,2 \times 1,1$ & $3,2 \times 2,1 \times 1,2$ & - & - \\
\hline 8 & $\mathrm{~F}$ & 38 & 205,0 & 114,0 & 8,5 & 1,6 & 2,0 & Negativo & Negativo & Negativo & $2,2 \times 1,2 \times 1,3$ & $2,1 \times 1,1 \times 1,1$ & - & - \\
\hline 9 & $F$ & 36 & 66,0 & 162,0 & 11,0 & 1,2 & 0,4 & Negativo & Negativo & Negativo & $2,0 \times 1,4 \times 1,2$ & $3,2 \times 1,4 \times 1,2$ & - & - \\
\hline 10 & $\mathrm{~F}$ & 29 & 490,0 & 162,0 & 8,7 & 1,4 & 1,8 & Negativo & Negativo & Negativo & $3,9 \times 1,2 \times 1,1$ & $4,0 \times 1,4 \times 1,4$ & - & - \\
\hline 11 & $F$ & 39 & 385,0 & 128,0 & 8,3 & 1,6 & 1,1 & Negativo & Negativo & Negativo & $3,7 \times 0,6 \times 0,8$ & $3,2 \times 0,6 \times 0,6$ & - & - \\
\hline 12 & M & 37 & 210,0 & 95,0 & 8,8 & 1,4 & 1,8 & Negativo & Negativo & Negativo & $3,4 \times 1,8 \times 1,6$ & $3,5 \times 2,0 \times 1,4$ & - & - \\
\hline 13 & $F$ & 56 & 129,0 & 106,0 & 8,2 & 1,6 & 1,1 & Negativo & Negativo & Negativo & $3,4 \times 1,4 \times 0,7$ & $3,5 \times 1,3 \times 0,7$ & - & - \\
\hline Média & & 39 & 262,31 & 118,62 & 8,70 & 1,32 & 1,50 & & & & & & & \\
\hline
\end{tabular}

T3: triiodotironina; T4; tiroxina; T4L: tiroxina livre; TSH: hormônio tireoestimulante; A-Tg: anticorpo antitireoglobulina; A-TPO: anticorpo antitireoperoxidase; TSHR: anticorpo antireceptor do TSH. 
Tabela 6. Descrição dos valores de tendência central e dispersão por grupo de estudo valores da iodúria $(\mu \mathrm{g} / \mathrm{L})$.

\begin{tabular}{lcc}
\hline Grupo & & Descrição \\
\hline 1 & Média & 203,50 \\
Hipertireodismo & Mediana & 159,50 \\
& Desvio-padrão & 152,71 \\
& Mínimo & 40,00 \\
& Máximo & 430,00 \\
\hline Hipotireodismo & Média & 258,31 \\
& Mediana & 240,00 \\
& Desvio-padrão & 148,20 \\
& Mínimo & 51,00 \\
3 & Máximo & 590,00 \\
Tireoidite & Média & 244,29 \\
crônica autoimune & Mediana & 125,00 \\
& Desvio-padrão & 191,60 \\
& Mínimo & 63,00 \\
Controles & Máximo & 570,00 \\
\hline Amiodarona & Média & 1157,50 \\
& Mediana & 1105,00 \\
& Mesvio-padrão & 261,80 \\
& Mínimo & 920,00 \\
& Máximo & 1500,00 \\
\hline & Média & 262,31 \\
& Mediana & 210,00 \\
& & 146,20 \\
& & 60,00 \\
& & 490,00 \\
\hline
\end{tabular}

\section{DISCUSSÃO}

Verificou-se neste estudo que a excreção de iodo na urina dos pacientes com tireopatias autoimunes de Santo André, SP, encontra-se dentro dos parâmetros estabelecidos como região de suficiência para a ingestão de iodo, quando comparados ao grupo-controle (média de $203,50 \mu \mathrm{g} / \mathrm{L}$ para hipertireoidismo, 258,31 $\mu \mathrm{g} / \mathrm{L}$ para hipotireoidismo, $244,29 \mu \mathrm{g} / \mathrm{L}$ para tireoidite crônica autoimune e $262,31 \mu \mathrm{g} / \mathrm{L}$ para o grupo-controle) (Tabelas 1 a 6). Apenas os pacientes que recebiam amiodarona apresentam iodúria em excesso (média de $1.157,50 \mu \mathrm{g} / \mathrm{L})$.

Sabe-se que a ingestão de iodo é muito variável ao redor da Terra, e estudos populacionais para avaliação da quantidade deste nutriente presente na alimentação são muito difíceis e imprecisos $(4,14,17)$. O Brasil é um país que apresentava enormes áreas deficientes em iodo por causa de condições geológicas, com importantes repercussões em termos de moléstias associadas à carência crônica de iodo, como bócio endêmico, prevalência aumentada de hipotiroidismo congênito e distúrbios neurológicos variados que causam dificuldade de aprendizado e evasão escolar $(4,14,18)$. Assim, tem sido necessário que se faça a suplementação do iodo na dieta por meio da adição do iodato de potássio ao sal de cozinha, conforme recomendação da Organização Mundial de Saúde $(4,18-21)$. Para avaliar se a população está recebendo o iodo por meio do sal, os inquéritos epidemiológicos empregam índices, dos quais os recomendados são a prevalência de bócio, verificada ao exame físico ou pela ultrassonografia e a determinação da excreção urinária de iodo, uma vez que a maior fonte de excreção de iodo é a urina e, assim, as dosagens urinárias permitem avaliar a suficiência da ingestão na dieta diária (18-21). Além disso, estudos populacionais comparativos indicam que há correlação entre os valores obtidos por amostra isolada de urina e as amostras de 24 horas $(12,15)$.

Por outro lado, o excesso de iodo na alimentação também pode ser deletério, e países como Japão e Estados Unidos, que apresentam ingestão de iodo elevada, algumas vezes acima de $1.000 \mu \mathrm{g} /$ dia, evidenciam maior prevalência de tireopatias autoimunes em regiões de maior oferta de iodo $(4,9,10,22,23)$. Os mecanismos pelos quais o excesso de iodo modularia a reação tireoidiana autoimune incluem toxicidade direta ao tireócito, imunogenicidade aumentada da tireoglobulina e efeitos diretos do iodo nas células do sistema imune (8-10). Quantidades elevadas de iodo são oxidadas pela enzima TPO, gerando elementos oxidativos que causam lesão da membrana celular e indução de processo inflamatório ou autoimune em indivíduos predispostos (8). Assim, a ingestão excessiva de iodo formaria moléculas de tireoglobulina mais iodada e mais imunogênica. Além disso, efeitos estimuladores do iodo nas células do sistema imune, como macrófagos, células $\mathrm{T}$, células dendríticas e células $\mathrm{B}$, podem exercer papel no desencadeamento da autorreatividade tireoidiana $(8,10)$.

Além do excesso de iodo pela ingesta, outra causa de excesso de iodo em um paciente é o tratamento com amiodarona, medicação largamente utilizada na prática clínica por ser eficiente no tratamento de grande número de arritmias cardíacas (24-26). Sua ação cardíaca é, em parte, ligada à bomba de $\mathrm{Na}^{+} \mathrm{K}^{+}$ATPase, mas, também, às alterações observadas no metabolismo dos hormônios tireoidianos. Apresenta dois átomos de iodo por molécula (37,5\% do peso molecular), porquanto $10 \%$ é desiodado em iodo livre (26); dessa forma, na dose de manutenção, de 200 a $600 \mathrm{mg} /$ dia, tem-se de 7 a $21 \mathrm{mg}$ de iodo livre, o que excede em muito as necessidades básicas diárias 
recomendadas (4). As complicações decorrentes do uso da amiodarona são o hipotireoidismo e a tireotoxicose, sendo esta última mais comum nas áreas carentes de iodo, enquanto o hipotireoidismo ocorre mais frequentemente nas áreas sem tal carência $(26,27)$. O hipotireoidismo é em razão do efeito de Wolff-Chaikoff, no qual a glândula tireóide apresenta bloqueio da organificação e ocorre em $6 \%$ a $13 \%$ dos pacientes, dependendo da carência de iodo na região (27). A tireotoxicose induzida pela amiodarona tem aparecimento súbito, que combinado com a doença cardíaca de base, gera situação normalmente muito grave (24-27). Este tipo de tireotoxicose pode ser dividido em tipo I, relacionado à existência de doença tireoidiana não expressa clinicamente, em especial, naquelas regiões carentes de iodo, onde o excesso de iodo contido na amiodarona irá promover a síntese aumentada de hormônios tireoidianos, fenômeno conhecido como Jod-Basedow; ou tipo II, resultado da ação citotóxica da amiodarona, que causa lesão ao tecido glandular com a consequente liberação dos hormônios da tireóide na circulação, fato que ocasiona a tireotoxicose.

Nos pacientes com tireopatias autoimunes avaliados neste estudo, o iodo na urina encontrava-se normal, com exceção do grupo amiodarona, demonstrando que esta é droga que pode desencadear hipotireoidismo em indivíduos geneticamente predispostos (26).

Recentemente, Camargo e cols. $(28,29)$, analisando a prevalência de tireoidite crônica autoimune na mesma área geográfica, atribuíram o aumento da prevalência da moléstia por aumento da ingesta de iodo. A interpretação do presente estudo é distinta; primeiramente, há necessidade de se investigar outros fatores ambientais para esclarecer o possível aumento da tireoidite crônica autoimune nos últimos anos e o que se sabe é que tem havido melhora significativa dos métodos diagnósticos para tireoidite autoimune, com a inclusão de testes mais sensíveis para a detecção de anticorpos e de ultrassonografia. Fato semelhante ocorreu com o aumento da incidência de câncer de tiróide nos Estados Unidos, causada pela melhora do diagnóstico dos nódulos por causa do uso extensivo da ultrassonografia, o que causou aumento do diagnóstico dos casos subclínicos ou anteriormente não-diagnosticados e não por elevação verdadeira na ocorrência da moléstia (30). Em segundo lugar, os dados do presente estudo, referentes às iodúrias de 1994 e de 2002, 2003 e em Santo André, não evidenciam aumento expressivo da ingestão de iodo na região, uma vez que as iodúrias dos escolares em 1994 e dos pacientes e controles incluídos neste estudo são equivalentes e revelam suficiência na ingestão de iodo.
A combinação de dois fatores, genético predisponente e ambiental, pode ser coparticipante no mecanismo desencadeante de tireopatias autoimunes e, assim, outros fatores ambientais devem ser investigados, já que de acordo com os resultados deste estudo, o iodo não pode ser considerado o agente responsável pelos casos de tireopatias autoimunes em regiões de divisa dos municípios de Santo André e São Paulo, estado de São Paulo.

Agradecimentos: Este trabalho foi financiado pela Fundação de Amparo à Pesquisa do Estado de São Paulo (Fapesp), Auxílio à Pesquisa $\mathrm{n}^{\circ}$ 06/5937-9. Os autores agradecem à Angela Maria Faria pela eficiente ajuda secretarial e aos pacientes pela colaboração e compreensão, sem os quais este trabalho jamais poderia ser realizado.

Declaração: Os autores declaram que neste manuscrito não há potencial conflito de interesse científico dos autores.

\section{REFERÊNCIAS}

1. Vanderpump MPJ, Tunbridge WMG, French JM, Appleton D, Bates D, Clark $F$, et al. The incidence of thyroid disorders in the community: a twenty-year follow-up of the Whickam Survey. Clin Endocrinol (Oxf). 1995;43:55-68.

2. Tunbridge WMG, Vanderpump, MPJ. Population screening for autoimmune thyroid disease. Endocrinol Metab Clin North Am. 2000;29:239-53.

3. Wang C, Crapo LM. The epidemiology of thyroid disease and implications for screening. Endocrinol Metab Clin North Am. 1997;26:189-218.

4. Knobel M, Medeiros-Neto G. Moléstias associadas à carência crônica de iodo. Arq Bras Endocrinol Metab. 2004;48:53-61.

5. Rapoport B, McLachlan SM. Thyroid autoimmunity. J Clin Invest 2001;108:1253-9

6. Weetman AP. Autoimmune thyroid disease: propagation and progression. Eur J Endocrinol. 2003;148:1-9.

7. Collins J, Gough S. Autoimmunity in thyroid disease. Eur J Nucl Med. 2002;29:S417-24.

8. 8. Sgarbi JA, Maciel RMB. Patogênese das doenças auto-imunes da tiróide. Arq Bras Endocrinol Metab. 2007; no prelo.

9. Rose NR, Rasooly L, Saboori AM, Burek CL. Linking iodine with autoimmune thyroiditis. Environ Health Perspect. 1999;107:749-52.

10. Prummel MF, Strieder T, Wiersinga WM. The environment and autoimmune thyroid diseases. Eur J Endocrinol. 2004;150:605-18.

11. Maciel RMB. O laboratório no diagnóstico e seguimento de doenças auto-imunes e neoplásicas de tireóide. Arq Bras Endocrinol Metab. 2002; 46:65-71.

12. Esteves RZ. Determinação da excreção urinária de iodo em escolares brasileiros [tese]. São Paulo: Universidade Federal de São Paulo; 1997.

13. Sandell EB, Kolthoff IM. Microdetermination of iodine by a catalytic method. Mikrochemica Acta. 1937;1:9-25.

14. Esteves RZ, Kasamatsu TS, Kunii IS, Furuzawa, GK, Vieira JGH, Macie RMB. Desenvolvimento de um método para a determinação da iodúria e sua aplicação na excreção urinária de iodo em escolares brasileiros. Arq Bras Endocrinol Metab. 2007;51:1477-84.

15. Dunn JT, Crutchfield HE, Gutekunst R, Dunn AD. Methods for measuring iodine in urine. International Council for Control of lodine Deficiency Disorders, Wageningen: ICCIDD; 1993.

16. Barros F, Victora C. Inquérito para avaliação da prevalência de bócio em escolares brasileiros. Epicentro: Universidade Federal de Pelotas; 1994. 
17. Als C, Minder C, Wellem D, Van Thi HV, Gerber H, Bourdoux P. Quantification of urinary iodine: a need for revised thresholds. Eur J Clin Nutr. 2003;57:1181-8.

18. Dunn JT. Seven deadly sins in confronting endemic iodine deficiency and how to avoid them. J Clin Endocrinol Metab. 1996;81:1332-5.

19. Hetzel BS. Eliminating iodine deficiency disorders-the role of the International Council in the global partnership. Bull World Health Organ. 2002;80:410-7.

20. lodine status worldwide: World Health Organization (WHO) Global Database on lodine Deficiency. ISBN 924 159200. Disponível em: http:// www.who.org.

21. International Council for Control of lodine Deficiency Disorders. Disponível em: http://www.iccidd.org.

22. Konno N, Makita H, Yuri K, lizuka N, Kawasaki K. Association between dietary iodine intake and prevalence of subclinical hypothyroidism in the coastal regions of Japan. J Clin Endocrinol Metab. 1994;78:393-7.

23. Pearce EN, Gerber AR, Gootnick DB, Khan LK, Li R, Pino S, et al. Effects of chronic iodine excess in a cohort of long-term American workers in West Africa. J Clin Endocrinol Metab. 2002;87:5499-502.
24. Silva JR, Guariento ME, Fernandes GA, Maciel RMB, Ward LS. Impact of long-term administration of amiodarone on the thyroid function of Chagas' disease patients. Thyroid. 2004;14:373-9.

25. Pavan R, Jesus AMX, Maciel LMZ. A amiodarona e a tireóide. Arq Bras Endocrinol Metab. 2004;48:176-82.

26. Camacho $\mathrm{CP}$, Maciel RMB. Hipotiroidismo/hipertiroidismo durante a terapia com amiodarona. In: Terapêutica Clínica das Doenças Cardiovasculares. São Paulo: FAH Fonseca. 2007.

27. Basaria S, Cooper DS. Amiodarone and the thyroid. Am J Med. 2005;118:706-14.

28. Camargo RYA, Tomimori EK, Neves SC, Knobel M, Medeiros-Neto G. Prevalence of chronic autoimmune thyroiditis in the urban area neighboring a petrochemical complex and a control area in São Paulo, Brazil. Clinics. 2006;61:307-12.

29. Camargo RYA, Tomimori EK, Neves SC, Rubio GS, Galrao AL, Knobel $M$, et al. Thyroid and the environment: exposure to excessive nutritional iodine increases the prevalence of thyroid disorders in Sao Paulo, Brazil. Eur J Endocrinol. 2008;159:293-9.

30. Davies $L$, Welch HG. Increasing incidence of thyroid cancer in the United States, 1973-2002. JAMA. 2006;295;2164-7. 